\title{
NANOTECHNOLOGY BASED TARGETED DRUG DELIVERY SYSTEMS IN BREAST CANCER THERAPY
}

\author{
Dulanga Weerathunga, Koshala Chathuri De Silva \\ BMS School of Science, Colombo, Sri Lanka
}

\begin{abstract}
Cancer is a highly heterogeneous and complex disease condition to understand because of its cellular and physiological systems. The most common cancer treatments are chemotherapy, radiotherapy and surgical excision. Out of them chemotherapy remains as the most widely used anti-cancer therapy. But unfortunately chemotherapy has plenty of drawbacks when considering its action on cancerous cells. Many chemotherapeutic drugs are highly toxic, not specific, poorly selective and less soluble because of these incidents cancer patients have severe side effects such as alopecia, renal failures, cardiac failures and etc. In order to overcome these problems in nanotechnology based targeted drug delivery system was introduced. Nanotechnology is a rapidly growing files which promote novel methods in cancer diagnosis, treatments and prognosis. In targeted drug delivery system use differently synthesized nanoparticles to reduce the drawbacks of chemotherapy. Nanoparticles such as liposomes, carbon nanotubes, virus mediated nanoparticles and nanodiamonds are some of the nanoparticles which are being discussed in this article. By using nanoparticles many of the drawbacks in conventional chemotherapy can be altered for an example by using surface functionalized nanoparticles chemotherapeutic drugs can be loaded into the particle and easily can be delivered to the exact tumor site without harming the non-cancerous cells while increasing the halflife of the drug. Likewise there are many advantages in nanotaenology based targeted drug delivery system over the conventional chemotherapy which will give a new ray of hope towards cancer patients to have a quality life.
\end{abstract}

Keywords: Nanotecnology, Targeted Drug Delivery,Nanoparticles, Active Targeting, Passive Targeting , Cancer

\section{Introduction}

Cancer can be defined as a highly heterogeneous complicated disease condition that incorporates a large set of disorders which may result in by continues indefinite growth (Jabir et al., 2012). Rapid proliferations of abnormal cells are one of the defining causes in cancer which may ultimately go beyond the limits. Cancer causes morbidity and mortality rates nearly with 14 million new cases and 8.2 million relate deaths every year in worldwide. Prostate, lung, colorectum stomach and liver cancers are the most common among men and in women breast, lung, colorectum, cervix and stomach cancers are the most common types (WHO.com, 2015). Genetic and phenotypic diversity of cancer define the complexity of this disease (Hare et al., 2016).

Out of all cancer incidents breast cancer remains as the most common cause of women cancer incidents and the second largest cause for the cancer relating deaths in the worldwide. Moreover breast cancer is hundred times common in women than men. Due to increased risk factors breast cancer incidents have been upsurge in the time of 1975 to 2000 and now one women in every eight is suffering from a breast cancer type. Late diagnosis of the breast cancer is associated with a poor prognosis and early detection of cancer will have a better prognosis due to advances that have happened in the treatment strategies (Assi et al., 2011; Eissemann et al., 2013).

\section{Types of Breast Cancers and Severity}

Origin of the breast cancer is associated with breast tissues especially from the inner lining of the milk ducts and lobules (Eissmen et al., 2013). For prognosis, treatments and prediction, immunohistochemistry markers are used to assess nodal involvement, tumour grade, tumour size, surgical margins and histologic type. Further, estragon receptor, human epidermal growth factor receptor 2, progesterone receptor which are considered as immune histo chemistry markers are generally used in order to categorize breast tumour into subtypes. The common breast tumour types include, 1) either oestrogen receptor or progesterone receptor positive where HER2 
is negative, 2) either progesterone receptor positive or oestrogen receptor positive where HER2 is positive, 3) oestrogen receptor and progesterone receptor negative where HER2 is positive (HER2 positive) and 4) all oestrogen, progesterone and HER2 are negative (triple negative). Normally, cancers with either progesterone receptor or oestrogen receptor positivity have higher prognosis when compared to cancers with both progesterone receptor and oestrogen receptor negativity (Dai et al., 2016).

\section{Treatments for Cancer}

Treatment for cancer has become a challenge due to the complexity of the disease. Novel antineoplastic modalities such as immunotherapy, gene therapy, photothermal therapy, photodynamic therapy and hormonal therapy were introduced to treat cancer over the last decade. These remedies were able to induce cell apoptosis, alter gene mutations in angiogenesis process and cell cycle and adjust the gene transcription and translation conversions. However radiotherapy, chemotherapy and surgical excision still remains as the first line treatments for cancer (Kumari et al., 2016; Piktel et al., 2016). Out of them chemotherapy is one of the major therapeutic approaches that had been made for anticancer treatment, due to its systemic effect, usefulness as an adjuvant therapy, and can used to treat metastatic cancers. In addition chemotherapy uses in palliative care as well. The ultimate goal of chemotherapy is to deliver the drugs with the desired dose by giving sufficient circulation time for the absorption with the required half-life, to the site of action while preventing the normal cells and perform the therapeutic response (Shi, Gustafson \& Mackay, 2014). However due to the side effects of chemotherapy, uses has become limited and unable to produce optimum efficacy (Zhang et al., 2011). Some of the major side effects facing during the chemotherapy are discussed below.

\section{Limitations of Conventional Chemotherapy}

Tumour resistance formation for drugs is one of the major disadvantage associated with chemotherapy in cancer. Tumour resistance mechanisms have several origins. ATP binding cassette known as ABC transporter pays an important role in drug resistance to several drugs. Mainly members of $\mathrm{ABC}(\mathrm{ABCC} 1, \mathrm{ABCB} 1$, and $\mathrm{ABCG} 2)$ family are expressed in different types of cancer and form the drug resistance to cancer (Liang et al., 2009). Multi drug resistance (MDR) is divided in to two main classes, secondary and adenosine triphosphate binding cassette base on their energy source (Leonessa, 2003). Possibly most widely considered ABC transporter is P glycoprotein (Pgp) which belongs to the ABC superfamily and use ATP to actively pump molecules in one direction across the lipid membrane. In cancer Pgp is over expressed and contributes to multi drug resistance by pumping chemotherapeutic drugs out of the cells. Because the Pgp transmembrane domain (TMD) is capable to identify chemically unrelated molecules and translocate them, mainly which are hydrophobic because $\mathrm{ABC}$ transporters permeates hydrophilic substances to pass through (Chang, 2003; Huang et al., 2016). More than $50 \%$ of cancer patients arises multi drug resistance. Furthermore drug resistance is may be due to intrinsic or extrinsic factor. At the beginning of the treatments intrinsic resistance is more common. Initially cancer cells are escaping from the exposure or repairing the damage and finally resistant cells survive and leads to acquired resistance. Both these pathways may initiate decrease drug accumulation, decrease drug activation, alter the drug targets and altered the gene expression. These may be found to be the drug barriers in MDR (Liang et al., 2009).

Delivering of the therapeutics agents in to the target site is one of the major challengers in the conventional chemotherapy (Kakde et al., 2011). Currently using drugs are distributed through the body via blood stream without preferential localization to the tumour area, while affecting the both malignant and normal cells which are rapidly dividing in the gut, bone marrow, lymphoid tissues, foetus and hail follicles (Piktel et al., 2016). For an example Epirubicin (EPI) and Anthracycline derivative are drugs which were used for hepatocellular carcinoma (HCC), had caused DNA destruction by disturbing the breakdown of the equilibrium and increasing of DNA topoisomerase II concentration covalent complexes. Therefore long term use of EPI was limited due to nonspecific toxicity to healthy cells. Mainly due to cardiac toxicity (Kumari et al., 2015).

Most of the prepared chemotherapeutic drugs have poor solubility in water and more hydrophobic (Dimendra $e t$ al., 2016).Therefore in order to avoid these conditions it is a necessity to produce drugs which can be administered intravenously. For an example paclitaxel in one of the most successful anticancer drugs in 1990s. 
However this drug was extremely hydrophobic with no water solubility $(0.5 \mathrm{mg} / \mathrm{L})$. The adjuvants which were used to solubilize the drug, including polyoxyethylate castor oil and dehydrated alcohol caused severe side effects like nephrotoxicity, cardiotoxicity, hypersensitivity reactions, hyperlipidaemia, abnormal lipoproteins patterns and erythrocyte aggregation (Kakde et al., 2011). Therefore in order to avoid the usage of adjuvants a new method of drug delivery was needed.

In addition chemotherapeutic drugs face lot of problems during transportation of the drugs to tumour sites. Physiochemical properties such as size, surface structure, and the charge plays an important role during drug delivery. Further due to heterogeneity of the cancer major difficulties are there when it comes to the drug delivery into whole tumour mass (Hare et al., 2016).

Likewise in the past few decades there were lot of problems in treating cancer patients with conventional chemotherapy. Therefore to overcome these difficulties scientists are investigating for new techniques.

\section{Nanotechnology in Cancer Biology}

In Nanotechnology materials and devices which less than $100 \mathrm{~nm}$ in size use for engineering, characterization and synthesis of various applications in many fields. Applications of nanotechnology in medicine have accelerated diagnosis, screening and treatments of many diseases (Diaz \& Vivas - Mejia, 2013). In cancer biology, nanotechnology can be used in various applications such as tumour detection, discovery of cancer biomarker and improvement of treatments.

Nanoparticles are small molecules that can be characterized as a whole unit in term of transport and properties (Tiwari et al., 2012; Prabhu et al., 2011). Nanoparticles in research filed has generated a passionate interest in scientists with the discovery of lipid based nanoparticles by Professor A.D. Bangham of the United Kingdom in 1960 (Puri et al., 2009).

Furthermore nanoparticles are the platform for effective and targeted drug delivery by overcoming many biological barriers with no or less side effects unlike the radiotherapy and chemotherapy. Furthermore nanotechnology based drug delivery system is a new method where target specifically for the cancer cells which have huge advantages over the conventional chemotherapy such as improvement in bioavailability and distribution of drugs ,longer shelf life, can administered with both hydrophobic and hydrophilic molecules through anywhere (oral, parental, nasal and intracellular routes) (Khanna, 2012). Therefore the new developments of nanotechnology based cancer therapies deliver a new ray of hope in the cancer research field (Calixto et al., 2016).

\section{Nanoparticle Drug Delivery System}

With the discovery of liposome, nanotechnology based targeted drug delivery system has made a significance impact on developing novel therapeutic approaches for many diseases as well as in cancer therapy (Puri et al., 2009; Shi et al., 2011). Different specialized nanoparticles such as liposomes, dendrimers, gold nanoparticles , silver nanoparticles, polymeric nanoparticles, carbon nano tubes, virus mediated nanoparticles, magnetic nanoparticles (table 1) and many other novel nanoparticles were introduced and will be introducing in the near future in to research field (figure 3)(Jabir et al., 2012). 


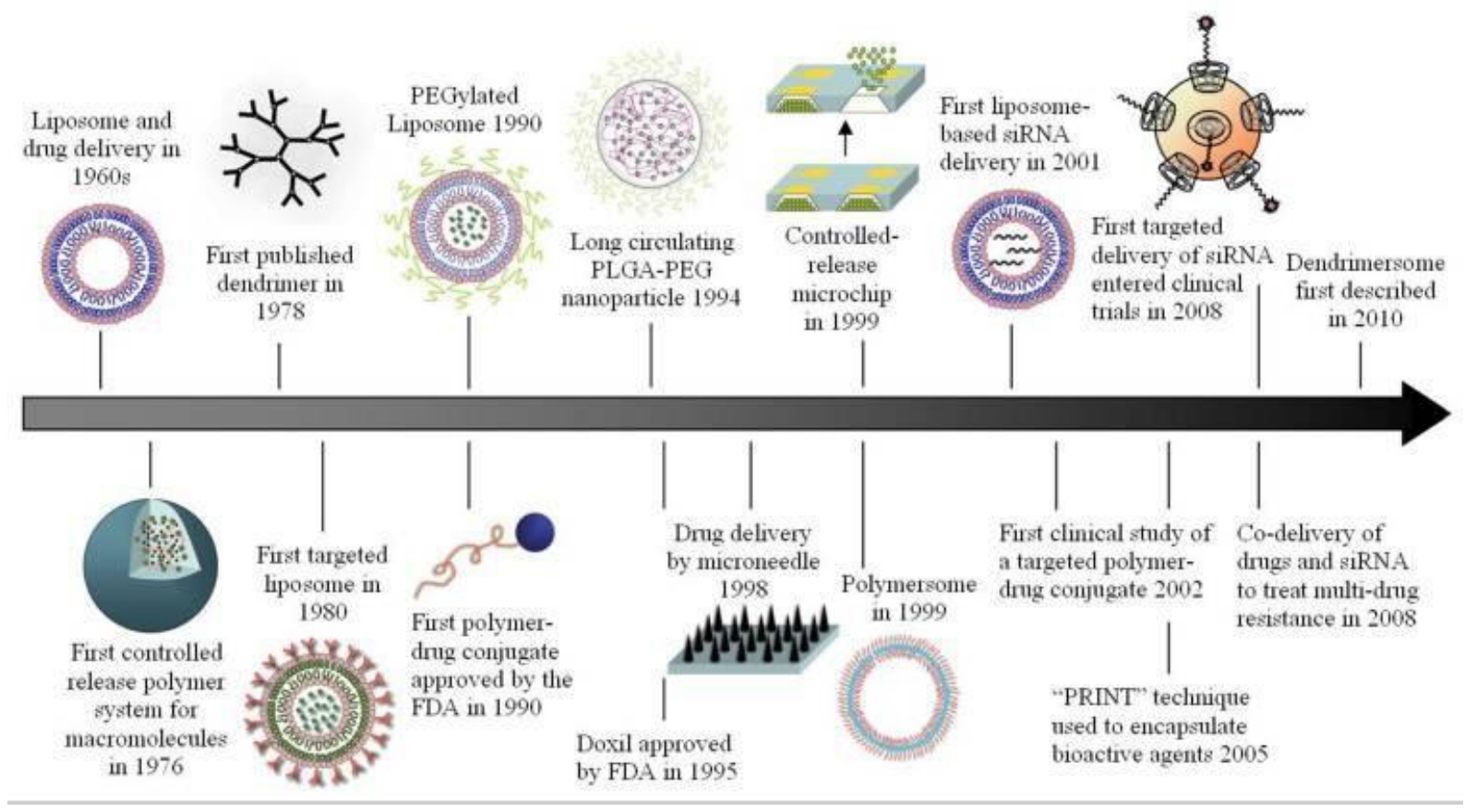

Figure 1: Development of nanotechnology based drug delivery (Shi et al., 2011)

Table 1 some of the nanoparticles with their characteristics associated with cancer therapies

\begin{tabular}{|c|c|c|}
\hline $\begin{array}{l}\text { Name of the } \\
\text { nanoparticle }\end{array}$ & Description & References \\
\hline $\begin{array}{l}\text { Lipid based } \\
\text { nanoparticles }\end{array}$ & $\begin{array}{l}\text { Liposomes are self-assembling and has a colloidal structure } \\
\text { consist with lipid bi layer. Outer most layer of the liposome } \\
\text { surrounds by a central aqueous area Via PEGylation, liposome } \\
\text { can be surface functionalized and can promote receptor- } \\
\text { mediated endocytosis by using targeting ligands such as } \\
\text { peptides, proteins, carbohydrates and antibodies and also } \\
\text { PEGyalation increase the circulation half-life while reducing the } \\
\text { clearance and immune recognition. These liposome are ideal } \\
\text { vehicles to deliver hydrophobic drugs without stimulating an } \\
\text { immune response. Furthermore liposomes are the most clinically } \\
\text { successful nanocarriers }\end{array}$ & $\begin{array}{l}\text { (Cho et al., 2008; } \\
\text { Lammers et al., } \\
\text { 2008; Miller, 2013). }\end{array}$ \\
\hline Nanodiamonds & $\begin{array}{l}\text { Nanodiamonds can be divided in to two main type fluorescent } \\
\text { nanodiamonds and detonation nanodiamonds. These particles are } \\
\text { led to applications because of their favourable properties such as } \\
\text { the facet surface possess electrical charge where the drug can be } \\
\text { attached to the neutral surface. And has electrostatic charge } \\
\text { allowing to disperse in the fluids. Furthermore nanodiamonds are } \\
\text { nontoxic cheap in production. Nanodiamonds have a greater } \\
\text { biocompatibility and stable and easy to purify. There are ongoing } \\
\text { researches on with doxorubicin attached nanodiamonds }\end{array}$ & ( Ho et al., 2015). \\
\hline $\begin{array}{l}\text { Virus mediated } \\
\text { nanocarriers } \\
\text { (VNPs) }\end{array}$ & $\begin{array}{l}\text { With a great efficiency viruses are able to infect naturally to a } \\
\text { host cell and delivery the payload of the genetic materials with } \\
\text { response to Ph changes, chemical stimuli, temperature and redox } \\
\text { status These phenomenon was taken to design an ideal virus }\end{array}$ & $\begin{array}{l}\text { (Ylidiz et } \text { al., 2011; } \\
\text { Van Kan-Danvelaar } \\
\text { et al., 2014; } \\
\text { Khodabandehloo et }\end{array}$ \\
\hline
\end{tabular}




\begin{tabular}{|c|c|c|}
\hline & $\begin{array}{l}\text { mediated nanocarriers. Plant based viruses are considered as the } \\
\text { safe delivery vehicles as they biocompatible, biodegradable, } \\
\text { nontoxic and less likely to trigger downstream effect in humans } \\
\text { Furthermore well characterized monodisperse structures which } \\
\text { can be produced in large quantities are some advantages of } \\
\text { VNPs. VNPs are one of the most advanced ad versatile } \\
\text { nanomaterial produced by nature because of the highly } \\
\text { symmetrical structure. The basic structure of the VNPs has an } \\
\text { internal cavity which can be filled with drugs quantum dots and } \\
\text { other particles and external surface consist of ligands for the cell } \\
\text { specific delivery }\end{array}$ & al., 2016). \\
\hline $\begin{array}{l}\text { Magnetic } \\
\text { nanoparticles }\end{array}$ & $\begin{array}{l}\text { Under an external magnetic field magnetic drug delivery system } \\
\text { can be work with loaded drugs to reach the tumour site in a more } \\
\text { localized way. Also they can be synthesized and functionalized in } \\
\text { different sizes and structures according the loading and targeting } \\
\text { molecules). Synthesizing of these have several strategies they } \\
\text { may have core shell structure where the core if formed either } \\
\text { magnetite or maghemeite and the shell is made up of cationic } \\
\text { materials such as, polyethylene oxide, poloxamers, silica, metals } \\
\text { such as gold, dextran, polyethylene glycol; (PEG) while } \\
\text { providing the functional groups to be attached via cross links in } \\
\text { order to minimize opsonisation and trigger the binding to drugs } \\
\text { and other molecules }\end{array}$ & $\begin{array}{l}\text { (Dobson, 2006; } \\
\text { Fang, 2009; Yang et } \\
\text { al., 2012; Mody et } \\
\text { al., 2014; Gobbo et } \\
\text { al., 2015). }\end{array}$ \\
\hline $\begin{array}{l}\text { Carbon } \\
\text { nanotubes }\end{array}$ & $\begin{array}{l}\text { These are huge cylindrical shape large molecules consist with a } \\
\text { hexagonal arrangement of sp } 2 \text { hybridized carbon atoms. The wall } \\
\text { is consist with multiple layers of grapheme sheets, formed by } \\
\text { rolling simple sheet. These are used to detect protein, DNA and } \\
\text { carriers to deliver vaccine. These are insoluble in all solvents. } \\
\text { Carbon nanotubes can be used to deliver drugs via intravenous } \\
\text { routes. }\end{array}$ & $\begin{array}{l}\text { (Cho et al., 2008; } \\
\text { Madani et al., 2011 } \\
\text { Zhang et al., 2011; } \\
\text { Kushwaha et al., } \\
\text { 2013). }\end{array}$ \\
\hline
\end{tabular}

Size and the surface characteristics are one of the most important features in nanoparticles. The size of the nanoparticle should be maintain in a tuneable manner which means their size need to be large enough to prevent leakage into blood vessels and small enough to escape from macrophages and penetrate into tumour site(Singh \& Lillard, 2009). In addition surface structure is important in determining the life span escape from macrophages. Therefore nanoparticles can be functionalized in order to achieve these characteristics with pegylation (Cho et al., 2008).

The development of targeted therapy systemically represent delivered nanoparticles reaching to the tumour tissues overcoming the obstacles with the minimal loss of volume and activity (Jin et al., 2014). Potential effect of nanoparticles describes by the different targeting strategies towards the tumour site (Kamal et al., 2012). Therefore delivery of nanoparticles into the tumour site can be presented in two major pathways, passive and active targeting.

\section{Passive Targeting}

Passive targeting can be mainly achieved through enhanced vascular permeability (EPR) effect (figure 3) (Piktel et al., 2016). 
EPR effect mediated the delivery of nanoparticles into the tumour site because of the defective structure of the blood vessels in order to ensure adequate supply of nutrients and oxygen to the tumour cells. Which means tumour angiogenesis is defective and characterized by leaky epithelium, impaired lymphatic drainage, and reduction in up taking the intestinal fluid. Furthermore consequent accumulation of the macromolecules provides a supportive environment to the passive targeting. With the accumulation of macromolecules, nanoparticles also get into the tumour site as a result of EPR effect (Piktel at el., 2016).

Properties of the nanoparticles such as size, circulation half-life, $\mathrm{pH}$ of the medium and surface nature are also important factors which need to be considered in passive targeting (Barkat et al., 2011).

\section{Active Targeting}

Active targeting is specially based on ligand - mediated or antibody mediated specific interactions between cancer cell surface and NPs (figure 4) (Zamboni et al., 2012; Jin et al., 2014; Wu et al., 2015). Furthemore this way of targeting is more specific and can be reduce the cytotoxicity to the normal cells (figure 4) (Jabir et al., 2012; Barkat et al., 2015).

This phenomenon is attaching homing ligands on the surface of the nanosystem and enabling active binding with receptors which are overexpressed in the cancer cells. There are a wide ranges of targeting molecule examples for ligand based targeted molecules which can be used as nanocarriers surface modifiers are peptides, proteins, folic acid, glycoproteins (transferrin), nucleic acids, monoclonal antibodies or their fragments and aptamers (short single stranded RNA or DNA EGFR, transferrin receptor and CD22 or CD44 are some of the receptors present in tumour cells. . Subsequently tumour specific ligands interact with the receptors in the cancer tissues and triggers the receptor mediated endocytosis and internalization of the nanocarriers into the tumour cell (Grodzinski, 2014).

\section{Nanoparticle Based Targeted Drug Delivery Systems for Breast Cancer in Clinical and Pre -Clinical Studies}

In order to develop advanced nanoparticle based drug delivery system several clinical and pre-clinical studies have been done and some of them are already approved by the FDA. Some of the clinical and pre-clinical studies which give evidence about the advancements of this system are given below.

Doxorubicin is an anthracycline anticancer drug which has been used as a chemotherapeutic agent for the treatments of several cancer types such as breast cancer, tissue sarcoma, ovarian cancer and leukaemia. Action of doxorubicin includes DNA intercalation, topoisomerase II inhibition, and lipid peroxidation. But free doxorubicin has fatal side effects such as alopecia, nephrotoxicity, cardiotoxicity and renal failures. Therefore in order to reduce the side effect doxorubicin was encapsulated into liposomes (figure 1). In 1995, liposomal based doxorubicin (DOX) was approved by the FDA. As shown in figure 1 myocet and Doxil is the first approved drugs. Drugs' pharmacokinetics shows elimination half-life of Dox is 55 hours and $900 \mu \mathrm{g} \mathrm{h} \mathrm{mL} \mathrm{m}^{-1}$ area under the plasma concentration, myocet 2.5 hours and $45 \mu \mathrm{g} \mathrm{h} \mathrm{m}^{-1}$ and elimination half - life of free doxorubicin is 0.2 hours $4 \mu \mathrm{g} \mathrm{h} \mathrm{mL} L^{-1}$ longest circulation time has Doxil when compared to free drugs (figure 3). This blood circulating time is increased in liposomes with the reduction of size, pegylated surface and with lipid bilayer. In a phase III clinical trial which was conducted to treat metastatic breast cancer had showed a comparison with DOXIL and myocet shows less cardiac toxicity, neutropenia, vomiting and alopecia (figure 2)(Chang and Yeh, 2011). 


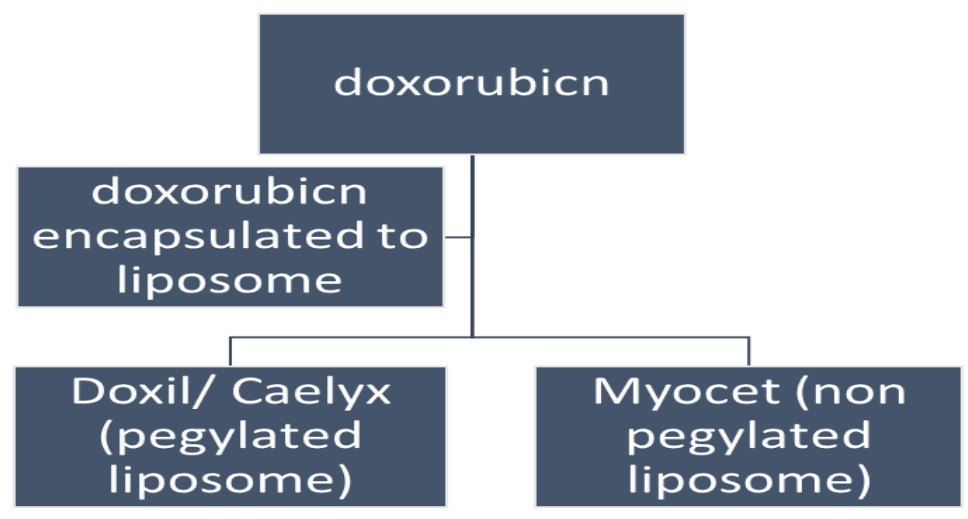

Figure 2: Encapsulation of the doxorubicin with liposomal nanoparticles

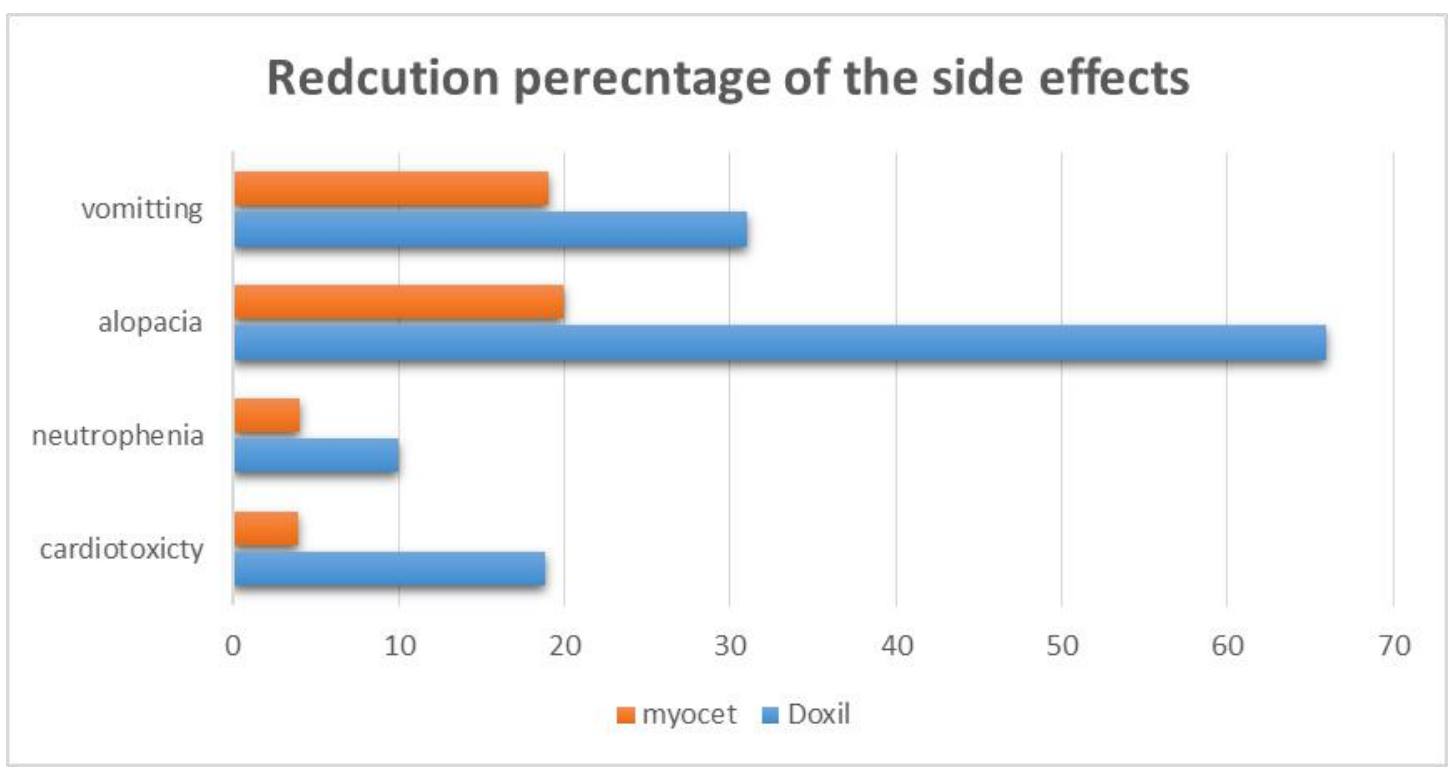

Figure 3: Reduction percentages of the side effects

Another study was conducted to target overexpressed HER-2 receptor by using liposomal doxorubicin. In this study pegylated liposomal was designed to target HER-2. The study was conducted using BT474 -M3 breast cancer cells which were inoculated to mice and had showed that HER-2 targeted liposomal doxorubicin has effectively been internalized into the tumour cells than free drug while inhibiting the tumour growth.

Another pre - clinical study has been done on integrin $\alpha v \beta 3$ is a subset of a tumour blood vessel which is associated with malignant tumour growth and angiogenesis. Therefore a nanoparticle was designed to target $\alpha v \beta 3$ by encapsulation doxorubicin (Dox). After synthesizing the targeted nanoparticle (RGD-NP) in order to address the specificity of the nanoparticle to integrin $\alpha v \beta 3$ binding studies were conducted using human umbilical vein endothelial cells (HUVECs), which has higher amount of integrin $\alpha v \beta 3$ with targeted peptides and controlled peptides and had shown a complete binding inhibition with control peptide and binding and internalization with the target peptide, which shows the advantage of active targeting strategy. Then RGD-NP targeting to the tumour vasculature were tested using M2IL-GFP mouse melanoma cells (integrin negative) and was allowed to get vascularized for 7 days. Then RGD -NP (targeted) and RAD -NP (none targeted) were injected and observed. Within 2 hours of injection RGD -NP targeted the newly formed vasculature in the margin of the tumour and reached maximum binding after 5 hours of injection, though RAD-NP was not accumulated in the tumour. After that in order to study anti-angiogenic action of the targeted nanoparticle mice which have stromal cells loaded with basic fibroblast growth factor on the flank and injected DOX ( $1 \mathrm{mg} / \mathrm{kg}$ total 
DOX) loaded nanoparticle. After 7 days angiogenesis was measured and showed $70 \%$ inhibition of angiogenesis. Therefore with the previously experimented targeting RGD-NP is an antagonist for integrin av $\beta 3$ and targeting of DOX loaded NP produce a strong anti angiogenic effect on vasculature (figure 4) (Murphy et al., 2008). Which proves that nanoparticles conjugated with DOX can be easily targeted via active targeting and does it action at the desired site of action.

However liposomal based nanoparticles has showed dose limiting side effects such as palmar plantar erythrodysesthesia (PPE) or hand foot syndrome small amounts of drug can leak into palms and soles of the feet and can be resulted in tenderness, redness and peeling of the skin (Rafiyath et al., 2012).

Another clinical study was conducted with Trastuzumab (TZ) which is a monoclonal antibody approved by the Food and Drug Administration. Combining TZ with other chemotherapeutics, taxanes and anthracyclines, has given significant improvement of disease-free survival rates.TZ-DTX (docetaxel) combination treatment had a greater chance of survival than free DTX alone and had a lower incidence of febrile neutropenia and symptomatic heart failure.

Cytotoxicity of the DTX-loaded dendrimers was significantly higher than free DTX. Targeted dendrimers were more effective than non-targeted ones.TZ-Dend-DTX was more cytotoxic than Dend-DTX due to the interaction between TZ and HER2 receptors and internalization of receptor-mediated endocytosis is more efficient.

One of the most significant breast cancer antitumor drug, Paclitaxel (PTX) has an action in microtubule assembly stimulation however prevents the microtubule disassemble. Therefore the hindrance of microtubule functions like cell transport, mitosis and cell motility occurs. The specificity of drug is less therefore this action affects all fast dividing cells. Thus causing many side effects. To avoid these side effects SWNT-HSA (single wall carbon nanotube with human serum albumin) nanoparticle was introduced. Due to high demands of oxygen and nutrients the vascular permeability has been enhanced in fast growing tissues like tumours. A main feature of tumour vasculature is a defective endothelium due to poor alignment. The EPR effect or passive targeting strategy allows the nano-particles to accumulate in the tumour tissue. Therefore passive targeting allows PTX a more targeted specific approach than free drug. An in vitro study was carried out using MCF-7 breast cancer cell lines to study the drug efficacy with SWNT-HSA and PTX alone. In here HSA has been used as an adapter to load PTX into SWNT. The percentage of apoptosis of the cells treated with free PTX, HSA/PTX and SWNTHSA/PTX was recorded (Figure 10). In comparison a gradual increase was identified proving the effective action of the nano-particles in the improvement of inducing apoptosis in tumour cells (Shao et al., 2013).

\section{Limitations of Nanotechnology Based Targeted Drug Delivery Systems and Future Perspective}

It is undebatable that nanotechnology offers various novel therapeutic approaches in drug targeting and delivering in anti-cancer therapies. But in some cases the enthusiasm need to be suppressed due to several reports on some limitations facing with nanotechnology based targeted drug delivery system. Accumulation of the nanoparticles and pharmacokinetic properties cannot be predicted easily by using their physicochemical properties (surface properties, size, and charge). Also when nanoparticles are consisting with resembling size and shape they are not strong enough to perform the desired task. Therefore there is an urge to develop a strategy to identify the exact properties of nanocarriers (Piktel et al., 2016).

Nanoparticles based cancer therapy may also responsible for several toxic effects too. Due to the small size these can easily penetrate into the healthy tissues and can cause damage also can be accumulated in kidneys without proper excretion. Regardless of the mobilization of cancerous agents, nanomaterials should improve biocompatibility as well. For an example administration of carbon nanotubes had been resulted in increasing the fibrotic reactions and inflammatory factors, by accumulation in airways (Piktel et al., 2016). Further as discussed earlier several nanoparticles such as liposomes tend to develop hand foot syndrome.

\section{Summary}

Nanotechnology is a rapidly growing filed while expanding its area in science. Also it plays an indispensable role in cancer biology therefore this drug delivery can be used specifically in breast cancer. This area of research is expected to develop novel, erudite and multifunctional applications in cancer diagnosing, treatments and 
prognosis. Researches on nanotechnology is vigorously aimed towards achieving cite specific targeting while overcoming the limitations in cancer treatment strategies especially chemotherapy. Nanotechnology based targeted drug delivery systems using functionalized nanoparticles give a new ray of hope towards chemotherapy based issues such as efficacy, safety, drug accumulation drugs induce toxicity and MDR. Clinical and preclinical studies pointed out that nanoparticles can be used for effective treatment with no or less side effects. Furthermore some of the nanotechnology based targeted drug delivery system are approved and are already being used in treatments. Therefore applications of nanotechnology based drug delivery systems in breast cancer treatment can be used as an advanced method of treating patient with cancer with minimum suffering. Several clinical and pre-clinical studies have showed that how this nanotechnology based targeted drug delivery has been effectively used in breast cancer therapy.

\section{References}

Assi, H., Khoury, K., Dbouk, H., Khalli, L., Mouhieddine, T., and Sagir, N. (2013) Epidemiology and prognosis of breast cancer in young women. journal of thoracic Disease, 5(1), 2-8.

Barakat, N., Talab, D., \& Salehi, A. (2011) Target Nanoparticles: An Appealing Drug Delivery Platform. Journal of Nanomedicine \& Nanotechnology, 4(1), 1-9.

Calixto, G., Fonseca-Santos, B., Chorilli, M., and Bernegossi, J. (2014) Nanotechnology-based drug delivery systems for treatment of oral cancer: a review. International Journal of Nanomedicine 9: 3719- 3735.

Chang, G. (2003) Multidrug resistance ABC transporters. FEBS Letters 555: 102- 105.

Cho, K., Wang, X., Nie, S., Chen, Z., and Shin, D. (2008) Therapeutic Nanoparticles for Drug Delivery in Cancer. Clinical Cancer Research, 14(5), 1310-1316.

Dai, X., Xiang, L., Li, T., and Bai, Z. (2016) Cancer Hallmarks, Biomarkers and Breast Cancer Molecular Subtypes. Journal of Cancer, 7(10),1281-1294.

Díaz, M. \& Vivas-Mejia, P. (2013) Nanoparticles as Drug Delivery Systems in Cancer Medicine: Emphasis on RNAi-Containing Nanoliposomes Pharmaceuticals,6 (11), 1361-1380.

Dimendra J Patel, J. (2016) Treatment of cancer by using Nanoparticles as a Drug Delivery International Journal of Drug Development and Research 4: 1-14.

Dobson, J. (2006) Magnetic nanoparticles for drug delivery Drug Delivery Research 67(1): 55-60.

Espelin, C., Leonard, S., Geretti, E., Wickham, T., and Hendriks, B. (2016) Dual HER2 Targeting with Trastuzumab and Liposomal-Encapsulated Doxorubicin (MM-302) Demonstrates Synergistic Antitumor Activity in Breast and Gastric Cancer. Cancer Research, 76(6), 1517-1527.

Fang, C. \& Zhang, M. (2009) Multifunctional magnetic nanoparticles for medical imaging applications. Journal of Materials Chemistry, 19(35), 6258.

Gobbo, O., Sjaastad, K., Radomski, M., Volkov, Y., \& Prina-Mello, A. (2015) Magnetic Nanoparticles in Cancer Theranostics. Theranostics 5 (11): 1249-1263.

Hare, J., Lammers, T., Ashford, M., Puri, S., Storm, G., \& Barry, S. (2016) Challenges and strategies in anticancer nanomedicine development: An industry perspective. Advanced Drug Delivery Reviews 1: 1-14.

Ho, D., Wang, C., \& Chow, E. (2015) Nanodiamonds: The intersection of nanotechnology, drug development, and personalized medicine. Science Advances 1: 1500439 -1500439.

Huang, Y., Cole, S., Cai, T., \& Cai, Y. (2016) Applications of nanoparticle drug delivery systems for the reversal of multidrug resistance in cancer (Review). Oncology Letters 12: 11-15. 
Jabir, N., Kamal, M., Tabrez, Ashraf, Shakil, \& Damanhouri, (2012) Nanotechnology-based approaches in anticancer research. International Journal of Nanomedicine 7: 4391-4408.

Jin, S., Jin, H., \& Hong, S. (2014) Targeted Delivery System of Nanobiomaterials in Anticancer Therapy: From Cells to Clinics. BioMed Research International 2014: 1-23.

Kakde, D., Deepti, J., Vivek, S., Rajendra, K., \& Patil, A. (2011) Cancer Therapeutics- Opportunities, Challenges and Advances in Drug Delivery. Journal of applied pharmaceutical science 1: 1-10.

Kamal, M., Jabir NR, N., Tabrez, Ashraf, Shakil, \& Damanhouri, (2012) Nanotechnology-based approaches in anticancer research. International Journal of Nanomedicine 7: 4391- 4391.

Khanna, V. (2012) Targeted Delivery of Nanomedicines. ISRN Pharmacology 2012: 1-9.

Khodabandehloo, H., Zahednasab, H., \& Ashrafi Hafez, A. (2016) Nanocarriers Usage for Drug Delivery in Cancer Therapy. Iran Journal of Cancer Prevention 9: 3966.

Kulhari, H., Pooja, D., Shrivastava, S., Kuncha, M., Naidu, V., Bansal, V., Sistla, R., and Adams, D. (2016) Trastuzumab-grafted PAMAM dendrimers for the selective delivery of anticancer drugs to HER2-positive breast cancer. Scientific Reports, 6(1).

Kumari, P., Ghosh, B., \& Biswas, S. (2015) Nanocarriers for cancer-targeted drug delivery. Journal of Drug Targeting 24: 179-191.

Kushwaha, S., Ghoshal, S., Rai, A., \& Singh, S. (2013) Carbon nanotubes as a novel drug delivery system for anticancer therapy: a review. Brazilian Journal of Pharmaceutical Sciences 49: 629-643.

Lammers, T., Hennink, W., \& Storm, G. (2008) Tumour-targeted nanomedicines: principles and practice. British Journal of Cancer 99 (3): 392-397.

Leonessa, F. (2003) ATP binding cassette transporters and drug resistance in breast cancer. Endocrine Related Cancer 10: 43-73.

Liang, X., Chen, C., Zhao, Y., \& Wang, P. (2009) Circumventing Tumor Resistance to Chemotherapy by Nanotechnology. Methods in Molecular Biology 21: 467-488.

MacKay, J., Shi, P., \& Gustafson, J. (2014) Genetically engineered nanocarriers for drug delivery.International Journal of Nanomedicine 9: 1617.

Miller, A. (2013) Lipid-Based Nanoparticles in Cancer Diagnosis and Therapy. Journal of Drug Delivery 2013 : $1-9$.

Mody, V., Cox, A., Shah, S., Singh, A., Bevins, W., and Parihar, H. (2013) Magnetic nanoparticle drug delivery systems for targeting tumor. Applied Nanoscience 4: 385-392.

Murphy, E., Majeti, B., Barnes, L., Makale, M., Weis, S., Lutu-Fuga, K., Wrasidlo, W., and Cheresh, D. (2008) Nanoparticle-mediated drug delivery to tumor vasculature suppresses metastasis. Proceedings of the National Academy of Sciences 105: 9343-9348.

Piktel, E., Niemirowicz, K., Wątek, M., Wollny, T., Deptuła, P., and Bucki, R. (2016) Recent insights in nanotechnology-based drugs and formulations designed for effective anti-cancer therapy. Journal of Nanobiotechnology, 14: 1-23.

Prabhu, V., Uzzaman, S., Grace, V., and Guruvayoorappan, C. (2011) Nanoparticles in Drug Delivery and Cancer Therapy: The Giant Rats Tail. Journal of Cancer Therapy 02: 325-334. 
Puri, A., Loomis, K., Smith, B., Lee, J., Yavlovich, A., Heldman, E., and Blumenthal, R. (2009) Lipid-Based Nanoparticles as Pharmaceutical Drug Carriers: From Concepts to Clinic. Critical Reviews in Therapeutic Drug Carrier Systems 26: 523-580.

Rafiyath, S., Rasul, M., Lee, B., Wei, G., Lamba, G., and Liu, D. (2012) Comparison of safety and toxicity of liposomal doxorubicin vs. conventional anthracyclines: a meta-analysis. Experimental Hematology \& Oncology, 1(1), 10 .

Shi, J., Votruba, A., Farokhzad, O., \& Langer, R. (2010) Nanotechnology in Drug Delivery and Tissue Engineering: From Discovery to Applications. Nanotechnology Letters 10: 3223-3230.

Singh, R. \& Lillard, J. (2009) Nanoparticle-based targeted drug delivery. Experimental and Molecular Pathology 86: $215-223$.

Tiwari, G., Tiwari, R., Bannerjee, S., Bhati, L., Pandey, S., Pandey, P., \& Sriwastawa, B. (2012) Drug delivery systems: An updated review. International Journal of Pharmaceutical Investigation 2: 2.

Van Kan-Davelaar, H., van Hest, J., Cornelissen, J., \& Koay, M. (2014) Using viruses as nanomedicines. British Journal of Pharmacology 171: 4001-4009.

Verma, S., Miles, D., Gianni, L., Krop, I., Welslau, M., Baselga, J., Pegram, M., Oh, D., Diéras, V., Guardino, E., Fang, L., Lu, M., Olsen, S., and Blackwell, K. (2012) Trastuzumab Emtansine for HER2-Positive Advanced Breast Cancer. New England Journal of Medicine, 367(19), 1783-1791.

W, S., A, P., L, R., and S, P. (2015) A New Carbon Nanotube-Based Breast Cancer Drug Delivery System: Preparation and In Vitro Analysis Using Paclitaxel. Cell Biology and Biophysics, 71(3), 1405-1414.

WHO (2012) International Agency for Research on Cancer, at: http://globocan.iarc.fr/Pages/online.aspx, World Health Organization visited, 1 January 2017.

WHO (2015) World Health Organization at: http://www.who.int/mediacentre/factsheets/fs297/en/, visited 1 January 2017.

Wu, T. and Zhou, S. (2015) Nanoparticle-Based Targeted Therapeutics in Head-And-Neck Cancer.International Journal of Medical Sciences 12: 187-200.

Yang, Y. and Wang, H. (2013) Perspectives of Nanotechnology in Minimally Invasive Therapy of Breast Cancer. Journal of Healthcare Engineering 4: 67-86.

Zamboni, W., Torchilin, V., Patri, A., Hrkach, J., Stern, S., Lee, R., Nel, A., Panaro, N., and Grodzinski, P. (2012) Best Practices in Cancer Nanotechnology: Perspective from NCI Nanotechnology Alliance. Clinical Cancer Research 18: 3229-3241.

Zhang, W., Zhang, Z., and Zhang, Y. (2011) The application of carbon nanotubes in target drug delivery systems for cancer therapies. Nanoscale Research Letter 6: 555. 